\title{
Inverse Dynamics and Power Consumption Model of Crab Motion of a Realistic Hexapod Robot
}

\author{
Abhijit Mahapatra, Shibendu Shekhar Roy, and Dilip Kumar Pratihar
}

\begin{abstract}
The present paper deals with a detail inverse dynamics and power consumption of a realistic hexapod robot with crab motion. The prescribed motion parameters necessary for the inverse dynamic analysis like displacement, velocity, acceleration of the joints are obtained from the kinematic analysis and motion planning of the hexapod robot. The foot ground interaction is considered as a point contact with zero impact velocity. The solution to the problem is not unique due to a highly redundant robotic system. An energy consumption model has been derived for statically stable wave-crab gaits after considering a minimum of the instantaneous power consumption of the robotic system for optimum feet forces. Minimum of power consumption is considered as the objective function with respect to linear equality and inequality constraints. The simulated results and discussions of the inverse dynamic analysis of the robotic system with crab motion on regular terrain are discussed.
\end{abstract}

Index Terms-Power consumption, inverse dynamics, hexapod robot, crab motion.

\section{INTRODUCTION}

Today with the advancement of technology, researchers have an urge to develop robotic vehicles that can maneuver and perform tasks like natural beings having legs. The reason is that legged robots have high degree of terrain adaptability and maneuverability. It can negotiate any kind of terrain by adjusting the lengths of its legs to maintain the desired body position and orientation during navigation. Hence, it has greater agility, omni-directional mobility compared to the wheeled or tracked robots. Therefore, it can operate in both structured and non-structured environments. However, coordination of various leg joints so as to produce the desired gait pattern and maintain stability during locomotion is extremely complex [1]. Considering these aspects, a six-legged robot is better suited than others for maneuverability over any kind of terrain. It has many advantages compared to two- or four-legged robots. It provides better static stability and is less susceptible to deadlock situations. The six-legged robot is more robust because it is able to walk with one or two failed legs, since it is possible to define stable gaits by using either four or five

Manuscript received January 5, 2015; revised April 24, 2015.

Abhijit Mahapatra is with the Virtual Prototyping \& Immersive Visualization Lab, CSIR-Central Mechanical Engineering Research Institute Durgapur, India (e-mail: abhi_mahapatra@ yahoo.co.in).

Shibendu Shekhar Roy is with the Department of Mechanical Engineering, National Institute of Technology, Durgapur, India (e-mail: ssroy99@yahoo.com).

Dilip Kumar Pratihar is with the Department of Mechanical Engineering, Indian Institute of Technology, Kharagpur, India (e-mail: dkpra@mech.iitkgp.ernet.in). legs. Also, compared to eight- or more-legged robots, its power consumption is less, since with the increase in the number of legs; a lot of actuators are to be controlled through a continuous coordination in addition to complicacies in kinematics and dynamics of legged mechanisms [2]-[5]. Therefore, a six-legged robot is a kind of optimal robotic structure to be used for varying terrains. But then, it requires a good control algorithm to achieve the mentioned cause, which means a suitable mathematical model of the complex six-legged robotic mechanism describing its kinematics and dynamic behavior during locomotion is necessary to develop.

The study of the legged robots by different researchers in the past as parallel manipulators shows that the models were simplified. It happens due to the complexities associated with the structure of the legged robots and their motion planning on varying terrains. Further, the followed approach to study of single leg dynamics was either by using classical Newton-Euler or Lagrange-Euler, and the study of kinematics corresponded to the use of Denavit-Hartenberg parameters setting or screw theory mostly [6]-[8]. Also, the models neglected the coupling effects and did not consider the inertia effects of the swing legs on the support legs and the trunk body. However, it is believed that a comprehensive dynamic model is crucial in the design, energy efficiency and control of legged robots. To achieve that, thorough understanding of the legged robot's locomotion is essential, which means that it is necessary to adopt the inverse dynamics approach [9]-[11].

The force distribution problem in legged robots is a constrained optimization problem. The issue had already been addressed in the literature by optimizing either feet forces or joint torques or power consumption [12]-[20]. But, the models were simplified by not taking into consideration the coupling effects, as discussed above. Moreover, to develop a good control algorithm, a good mathematical model of the complex multi-legged robotic mechanism describing the kinematics and dynamic behavior is necessary, so that the model will be able to address the above mentioned issues and analyze energy consumption of legged robots. The present study is about the development of an energy efficient inverse dynamic model of the hexapod robotic system for statically stable wave-crab gait by taking into consideration the coupling effects. The kinematic motion parameters (namely, displacement, velocity and accelerations) obtained from the inverse kinematic analysis (classical approach) of the robotic system with specified path and gait planning for crab motion in varying terrains are substituted in the inverse dynamic model, which is a set of algebraic equations. The equations are solved to determine the joint torques and resulting reaction forces for the feet in contact with the 
ground that are responsible to generate the prescribed motion trajectories. Optimization methodology like linear programming is used to solve for the feet forces and joint torques. A naturalistic objective function imposed is, minimization of power consumption for calculating contact foot-force distribution and determination of joint torques. The function is constructed as a summation of instantaneous power consumption for all the joints, and the problem is formulated such that it is minimized with respect to linear equality and inequality constraints.

The paper is divided into four sections. The preceding Section II describes the CAD model of the hexapod robot and mathematically defines the problem, in addition to the description of the optimization problem which is basically a linear programming method used as a basis for the solution. In Section III, the simulated results are discussed followed by a conclusion in Section IV.

\section{KINEMATIC AND DyNAMIC MODELING}

Fig. 1 shows a realistic CAD model developed in CATIA V5 with specific dimensions and subsequently, the mathematical model is developed to carry out both kinematic as well as dynamic analysis of the robotic system. The essential parameters that are obtained from the CAD model are listed in Table I. The main body-parts of the robot are made of aluminum. The total mass of the robot without payload is estimated to be equal to $2.456 \mathrm{Kg}$.

TABLE I: KINEMATIC AND DYNAMIC PARAMETERS OF THE ROBOT

\begin{tabular}{|c|c|c|c|c|c|}
\hline \multirow[b]{2}{*}{ Parameters } & \multicolumn{5}{|c|}{ Values } \\
\hline & $\begin{array}{l}\text { Trunk } \\
\text { body }\end{array}$ & Payload & $\begin{array}{l}\text { Link } i 1 \\
(i=1 \text { to } 6)\end{array}$ & $\begin{array}{l}\text { Link } i 2 \\
(i=1 \text { to } 6)\end{array}$ & $\begin{array}{l}\text { Link } i 3 \\
(i=1 \text { to } 6)\end{array}$ \\
\hline Mass (Kg) & 0.650 & $4.244^{\mathrm{a}}$ & 0.150 & 0.041 & 0.110 \\
\hline \multirow{3}{*}{$\begin{array}{l}\text { Mass } \\
\text { Moment of } \\
\text { Inertia } \\
\left(\mathrm{Kg}-\mathrm{m}^{2}\right)\end{array}$} & 0.016653 & 0.009 & 0.000071 & 0.000020 & 0.000098 \\
\hline & 0.002518 & 0.003 & 0.000108 & 0.000087 & 0.000087 \\
\hline & 0.016897 & 0.011 & 0.000057 & 0.000100 & 20.777 \\
\hline Length (m) & 0.495 & 0.150 & 0.085 & 0.120 & 0.100 \\
\hline
\end{tabular}

It is to be noted that each of the legs has three motorized revolute joints with angular variables, $\theta_{i 1}-\beta_{i 2}-\beta_{i 3}$ corresponding to local axes configuration $Z-Y-Y$, respectively, as shown in Fig. 1.

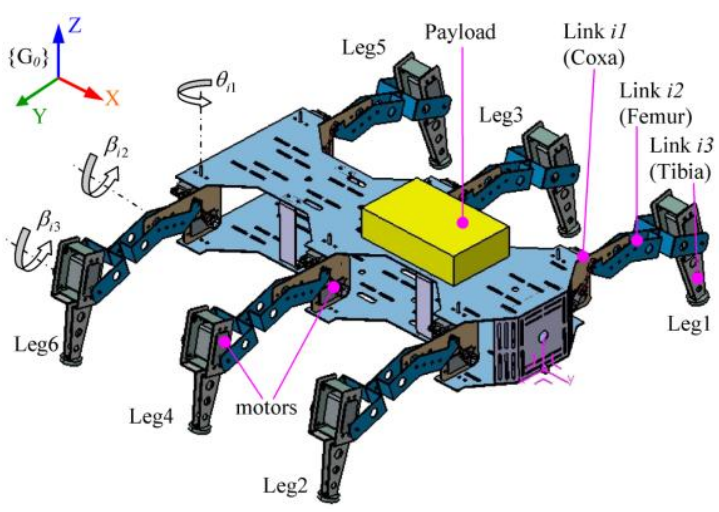

Fig. 1. A realistic hexapod robot (3D CAD model).
Therefore, the hexapod robot can be considered as robotic system having mobility levels of twenty four, out of which six correspond to the trunk body and eighteen are related to the legs. The system has been generalized by using orientation vector of Bryant angles [21], $\eta_{G}=\left[\alpha_{G} \beta_{G} \theta_{G}\right]^{T}$ with respect to global fixed frame $G_{0}$.

\section{A. Kinematic Modeling}

The hexapod robotic system is considered as a rigid multibody system with multiple reference frames (both with respect to global and local frames) for kinematic modeling, as shown in Fig. 2.

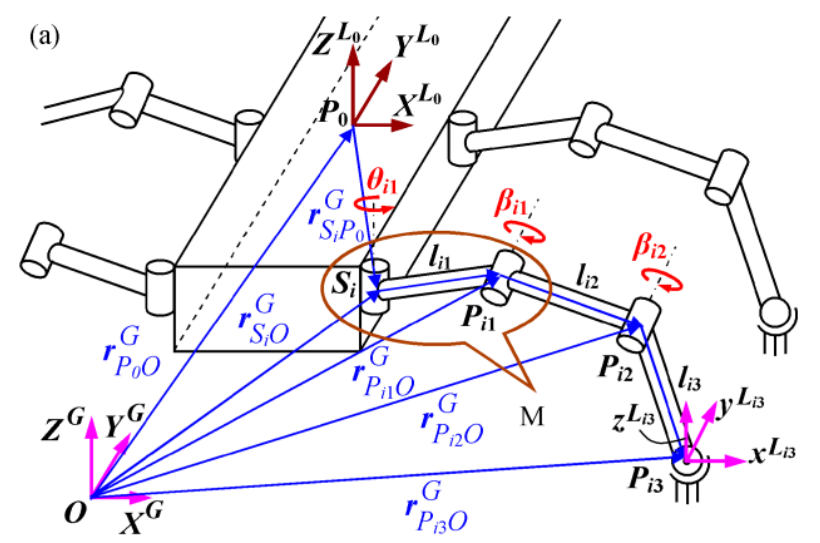

(b)

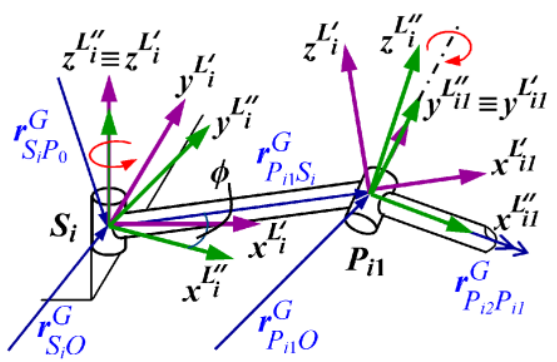

Fig. 2. Reference frames and vector diagram of the robotic system (a) Isometric view, (b) View ' $\mathrm{M}$ '.

Here, $G_{0}$ (shown in Fig. 1) and $\boldsymbol{G}$ (shown in Fig. 2) are the static and dynamic global reference frames, respectively, located at origin $O . L_{0}$ is the body fixed reference frame located at point $\mathrm{P}_{0}$ on the trunk body. $L_{i}^{\prime}, L_{i}^{\prime \prime}$ are the local frames of the successive joint states, respectively located at $S_{i}$. Similarly, the local frames: $L_{i j}^{\prime}, L_{i j}^{\prime \prime}$ are located at $P_{i j}$, where $j=1$ to 2 . $L_{i 3}$ is the tip point reference frames located at $P_{i 3}$. Also, $r_{P_{0} O}^{G}, r_{S_{i} O}^{G}, r_{P_{i j} O}^{G}(j=1$ to 3$)$ are the displacement vectors from point $O$ to $P_{0}, S_{i}, P_{i j}$, respectively represented in global reference frame $\boldsymbol{G}$. Here, $\phi$ is twisted angle of the coxa (in the present study $\phi=0)$. The vectors of cartesian coordinates of $P_{0}, \quad P_{i j}$ with respect to $G$ are represented by $p_{0}^{G}=\left(r_{P_{0} O}^{G}, \eta_{0}\right)^{T} \in R^{6} \quad, \quad p_{i j}^{G} \quad=\left(r_{P_{i j} O}^{G}, \eta_{i j}\right)^{T} \in R^{108}$, respectively, where $r_{P_{0} O}^{G}=\left[\begin{array}{lll}x_{P_{0} O}^{G} & y_{P_{0} O}^{G} & z_{P_{0} O}^{G}\end{array}\right]^{T}, \eta_{0}=\left[\begin{array}{ll}\alpha_{0} \beta_{0} & 0\end{array}\right.$ $\left.\theta_{0}\right]^{T}, r_{P_{i j} O}^{G}=\left[\begin{array}{lll}x_{P_{i j} O}^{G} & y_{P_{i j} O}^{G} & z_{P_{i j} O}^{G}\end{array}\right]^{T}, \eta_{i j}=\left[\begin{array}{lll}\alpha_{i j} & \beta_{i j} & \theta_{i j}\end{array}\right]^{T}$.

\section{1) Forward kinematics}

The set of kinematic constraint equations that governs the state of the trunk body and the $i^{\text {th }}$ leg at any instant of time are shown in (1). 


$$
g_{i}\left(p_{i}\right)=\left(\begin{array}{c}
g_{1}\left(p_{0}\right) \\
g_{2}\left(p_{0}\right) \\
g_{3 i}\left(p_{i}\right) \\
g_{4 i}\left(p_{i}\right) \\
g_{5 i}\left(p_{i}\right) \\
g_{6 i}\left(p_{i}\right) \\
g_{7 i}\left(p_{i}\right) \\
g_{8 i}\left(p_{i}\right) \\
g_{9 i}\left(p_{i}\right)
\end{array}\right) \equiv\left(\begin{array}{c}
r_{P_{0} O}^{G}-\left.r_{P_{0} O}^{G}\right|_{t=t_{0}}-\int f(t) \\
\eta_{0}-\left.\eta_{0}\right|_{t=t_{0}}-\int g(t) \\
r_{P_{0} O}^{G}+A^{G L_{0}} r_{S_{i} P_{0}}^{L_{0}}+A^{G L_{i}^{\prime \prime}} r_{P_{i 1}^{\prime \prime} S_{i}}^{L_{i}^{\prime \prime}}-r_{P_{i 1} O}^{G} \\
P_{r}^{T}(x, y) \cdot A^{L_{i}^{\prime \prime} G} \cdot A^{G L_{i}^{i}} \cdot P_{\mathrm{r}}(z) \\
r_{P_{11} O}^{G}+A^{G L_{i 1}^{\prime \prime}} r_{P_{i 2} P_{i 1}^{\prime \prime}}^{L_{i 1}}-r_{P_{i 2} O}^{G} \\
P_{r}^{T}(x, z) \cdot A^{L_{i 1}^{\prime \prime} G} \cdot A^{G L_{i 1}^{\prime}} \cdot P_{\mathrm{r}}(y) \\
r_{P_{i 2} O}^{G}+A^{G L_{i 2}^{\prime \prime}} r_{P_{i 3} P_{i 2}}^{L_{h}^{\prime}}-r_{P_{i 3} O}^{G} \\
P_{r}^{T}(x, z) \cdot A^{L_{i 2}^{\prime \prime} G} \cdot A^{G L_{i 2}^{i}} \cdot P_{r}(y) \\
r_{P_{i 3} O}^{G}-r_{i}^{G}
\end{array}\right)=\left(\begin{array}{c}
O_{3} \\
O_{3} \\
O_{3} \\
O_{2} \\
O_{3} \\
O_{2} \\
O_{3} \\
O_{2} \\
O_{3}
\end{array}\right)
$$

In (1), $P_{r}$ denotes the matrix projectors, $f(t)$ and $g(t)$ are the functions that govern the motion of the trunk body at any instant of time. $r_{i}^{G}$ is the coordinates of the tip point of link $l_{i 3}$ at any instant of time for both the stance and swing phases of leg $i$ due to gait planning and motion planning. The local components of $S_{i}, P_{i 1}, P_{i 2}$ and $P_{i 3}$ are fixed for a robotic structure and are given by $r_{S_{i} P_{0}}^{L_{0}}, r_{P_{i 1} S_{i}}^{L_{i}^{\prime \prime}}, r_{P_{i 2} P_{i 1}}^{L_{i 1}^{\prime \prime}}, r_{P_{i 3} P_{i 2}}^{L_{i 2}^{\prime \prime}}$, respectively. Also, $A^{G L_{0}}, A^{G L_{i}^{\prime}}, A^{G L_{i}^{\prime \prime}}, A^{G L_{i 1}^{\prime \prime}}, A^{G L_{i 2}^{\prime}}$, $A^{G L i 2}$ are the transformation matrices related to different local frames with respect to frame $G . A^{L_{i}^{\prime \prime} G}, A^{L_{i 1}^{\prime \prime} G}, A^{L_{i 2}^{\prime \prime} G}$ are orthogonal matrices of $A^{G L_{i}^{\prime \prime}}, A^{G L_{i 1}^{\prime \prime}}, A^{G L_{i 2}^{\prime \prime}}$, respectively. The functions are regulated by cubic polynomials, which are basically step functions given by the following expression [22]:

$$
h=h_{a}+a \cdot \Delta^{2}(3-2 \Delta) \text {, for } t_{a} \text { to } t_{b}
$$

where

$$
\begin{gathered}
a=h_{b}-h_{a} \\
\Delta=\left(t-t_{a}\right) /\left(t_{b}-t_{a}\right)
\end{gathered}
$$

$h_{a}$ represents the initial step value at time $t_{a}, h_{b}$ denotes the final step value at time $t_{b}$.

\section{2) Inverse kinematics}

To compute the power consumed by the hexapod during locomotion, it is necessary to calculate the joint position, velocity and acceleration. The solutions to the inverse kinematic problem for a single leg of the walking hexapod in support phase are given by the following expressions:

$$
\begin{aligned}
& \theta_{i 1}=\gamma-2 n \pi-2 \tan ^{-1}\left(\left(k_{i 1} \pm k_{i 4}\right) /\left(d_{i}+k_{i 2}\right), n \in \mathrm{I}\right. \\
& \beta_{i 2}=\phi-2 n \pi-2 \tan ^{-1}\left(\left(k_{i 6} \pm k_{i 7}\right) / k_{i 8}\right), n \in \mathrm{I} \\
& \beta_{i 3}=2 n \pi \pm 2 \tan ^{-1} \sqrt{ }\left(\left(1-k_{i 5}\right) /\left(1+k_{i 5}\right)\right), n \in \mathrm{I}
\end{aligned}
$$

where

$$
d_{i}=d_{i 1}+d_{i 2}-d_{i 3}
$$

$$
k_{i 1}=-\left[a_{i}\left(\mathrm{c} \beta_{0} \mathrm{c} \theta_{0}\right)+b_{i}\left(\mathrm{c} \alpha_{0} \mathrm{~s} \theta_{0}+\mathrm{s} \alpha_{0} \mathrm{~s} \beta_{0} \mathrm{c} \theta_{0}\right)+c_{i}\left(\mathrm{~s} \alpha_{0} \mathrm{~s} \theta_{0}-\mathrm{c} \alpha_{0} \mathrm{~s} \beta_{0} \mathrm{c} \theta_{0}\right)\right]
$$

$$
\begin{gathered}
k_{i 3}=-\left[-a_{i}\left(\mathrm{~s} \beta_{0}\right)-b_{i}\left(\mathrm{~s} \alpha_{0} \mathrm{c} \beta_{0}\right)+c_{i}\left(\mathrm{c} \alpha_{0} \mathrm{c} \beta_{0}\right)\right] \\
k_{i 4}=\sqrt{ }\left(k_{i 1}^{2}+k_{i 2}^{2}-d_{i}^{2}\right) \\
k_{i 5}=\left[\left(k_{i 3}-l_{i 1} \mathrm{~s} \phi\right)^{2}+\left(k_{i 4}-l_{i 1} \mathrm{c} \phi\right)^{2}-l_{i 2}^{2}-l_{i 3}^{2}\right] / 2 l_{i 2} l_{i 3} \\
k_{i 6}=k_{i 3}-l_{i 1} \mathrm{~s} \phi \\
k_{i 7}=\sqrt{ }\left[\left(k_{i 3}-l_{i 1} \mathrm{~s} \phi\right)^{2}+\left(k_{i 4}-l_{i 1} \mathrm{c} \phi\right)^{2}-\left(l_{i 2}+l_{i 3} k_{i 5}\right)^{2}\right] \\
k_{i 8}=l_{i 2}+l_{i 3} k_{i 5}+k_{i 4}-l_{i 1} \mathrm{c} \phi
\end{gathered}
$$

To compute the swing phase angles, it is necessary to substitute $k_{i 1}^{\prime}=-k_{i 1}, \quad k_{i 2}^{\prime}=-k_{i 2}$ and $k_{i 3}^{\prime}=-k_{i 3}$. The evaluation of joint angles with respect to time leads to the determination of the kinematic motion parameters like velocity, acceleration, trace of aggregate centre of mass etc. for a specified gait and motion planning of the robot on varying terrains. The parameters are subsequently transformed to global static reference frame $G_{0}$. Therefore, the velocity and acceleration vectors of the links $i 1, i 2$ and $i 3$ of $i^{\text {th }}$ leg with respect to $G_{0}$ are represented by $v_{i j}^{G_{0}}=\left(r_{P_{i j} O}^{G_{0}}, \omega_{L_{i j}^{\prime} G_{0}}^{L_{i j}^{\prime}}\right) \in R^{108}, \quad v_{i j}^{G_{0}}=\left(r_{P_{i j} O}^{G_{0}}, \omega_{L_{i j}^{\prime} G_{0}}^{L_{i j}^{\prime}}\right) \in R^{108}$ where $i=1$ to $6, j=1$ to 3 . During locomotion, the trunk body should have an uninterrupted and continuous motion for the given initial position, orientation (roll, pitch and yaw) and maximum velocity of the trunk body.

\section{3) Trajectory planning}

Trajectory planning of the swing legs is a computationally complex process. It is a fundamental step in the study of kinematics and dynamics of a six-legged robot. It is to be remembered that in the joint space, only the initial (home) and final (destination) positions are the matter of interest and therefore, is a case of point-to-point movement of joints. But, if a legged robot has to maneuver on a varying terrain and simultaneously, overcomes obstacles that come on its path of swing, it is required to define trajectory tracking in $3 \mathrm{D}$ cartesian space along which the tip of swing leg should traverse. Finally, the kinematic outputs are converted into some forms of joint commands of the robot, which means actuation of the joints. Here, in case of crab motion the crab angle $\left(\theta_{c}\right)$ is kept fixed for a given gait cycle. The absolute range of $\theta_{c}$ for the present robotic system is limited to $60^{\circ} \leq \theta_{c}$ $\leq 90^{\circ}$. Moreover, it is assumed that the full swing stroke of swing leg $i\left(s_{w}^{f}\right)$ is twice the swing stroke $\left(s_{0}\right)$ of the trunk body.

4) Gait planning

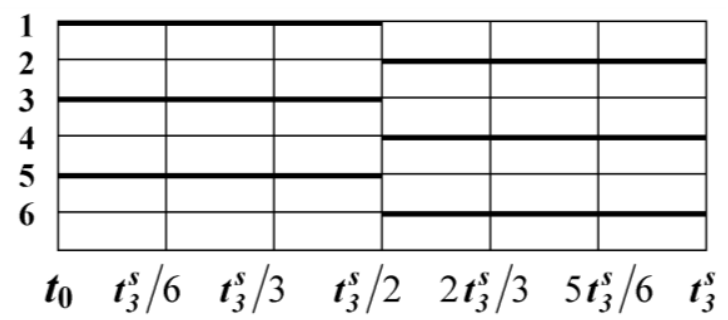

Fig. 3. Gait diagram $(D F=1 / 2)$. 
To move the robot's legs in a synchronous manner, it is necessary to have an effective gait planning and an efficient algorithm to address the movement of the trunk body and legs walking. In the present study, focus has been made on the wave-crab gait strategies with duty factor $(D F)=1 / 2$ (refer to Fig. 3) for locomotion along with the total gait cycle time, swing phase time and support phase time [1]. The total time of motion of the robot is equivalent to the time taken to complete $n$ duty cycles, i.e., $\left.t_{3}^{s}\right|_{n}=t_{3}$ (suffix $s$ represents swing).

\section{B. Inverse Dynamic Modeling}

In the present problem, a specified motion for a mechanical system is sought and the objective is to determine the forces that must act on the system to produce such a motion. This process is usually referred to as inverse dynamic or kinetostatic analysis.

The implicit constrained dynamic equation of the robotic system is given by the expression:

$$
M(p) . v={ }^{c} f+f(p, v)+q_{G C}(p, v) \in R^{114}
$$

where ${ }^{c} f \in R^{114}$ being the vector of constant reaction forces and torques of the joints associated with system coordinates denoted by $\left(g_{p}(p) . T(p)\right)^{T} . \lambda ; f(p, v) \in R^{114}$ is the vector of both known and unknown applied forces and torques; $q_{G C}(p$, $v) \in R^{114}$ is the vector of centrifugal forces and gyroscopic terms; $g_{p}(p) . T(p) \in R^{n_{c}, 114}$ is the constraint jacobian matrix; $\lambda \in R^{n_{c}}$ is vector of the lagrange multipliers; $M(p) \in R^{114,114}$ is the combined mass matrix of the robotic system.

The implicit constraint dynamic equation (17) is expressed in over complete cartesian coordinates $(p)$, which is often undesirable, since handling of the large number of equations is cumbersome and computationally intensive. Therefore, the kinematic motion parameters in cartesian space are transformed by kinematic transformation to the joint space [23] in terms of generalized coordinates $(q)$. The transformation of the dynamical model in the joint space is realized through explicit constraints given by the kinematic function,

$$
p=h(q)
$$

where $q \in\left[r_{P_{0}}^{G}, \eta_{0}, \theta_{11}, \beta_{12}, \beta_{13}, \theta_{21}, \beta_{22}, \beta_{23}, \theta_{31}, \beta_{32}, \beta_{33}, \theta_{41}\right.$, $\left.\beta_{42}, \beta_{43}, \theta_{51}, \beta_{52}, \beta_{53}, \theta_{61}, \beta_{62}, \beta_{63}\right]^{\mathrm{T}} \in R^{24}$,

The vector of velocity constraints of the system, both in cartesian space and joint space are related by the expression:

$$
v=J u
$$

Differentiating (19) with respect to time gives the expression of the acceleration constraints of the system given by the expression: where $J \in R^{114,24}$ is the jacobian matrix of the system in terms of generalized coordinates $(q)$ related to ground reaction forces and coupled joint torques.

It is to be noted that multiplication of the term ${ }^{c} f$ by Jacobian matrix $J$ leads to the elimination of the constraint,

$$
J^{c} f=0
$$

Hence, the transformation of the dynamic model in the joint space is realized by considering the following relation:

$$
D(q) u+C(q, q)=\tau \in R^{24}
$$

where $D(q)$ is the coupled mass and inertia matrix of the robotic system in terms of generalized coordinates, $\tau$ represents vector of ground reaction forces and coupled joint torques.

Also,

$$
\begin{gathered}
D(q)=J^{T} M(p) J \in R^{24 \times 24} \\
C(q, q)=J^{T} M(p) J u \in R^{24} \\
\tau=J^{T}\left[f(p, v)+q_{G C}(p, v)\right] \in R^{24}
\end{gathered}
$$

Twenty four number of equations are obtained in terms of ground reaction forces and coupled joint torques by substituting the necessary input values in (22). The equations are further arranged as described in the following paragraph.

The first set of six equations of (22) defines the static equilibrium of the trunk body and payload (combined) at any instant of time with respect to the forces and moments such that,

$$
\begin{gathered}
\sum_{i=1}^{6} F_{i}+F_{e}=O_{3} \in R^{3 \times 1} \\
\sum_{i=1}^{6}\left(r_{i} \times F_{i}\right)+M_{0}+M_{e}=O_{3} \in R^{3 \times 1}
\end{gathered}
$$

where $F_{e}$ and $M_{e}$ are the vectors representing the coriolis, centripetal, gravitational forces and moments acting on the trunk body and payload (combined), respectively, $F_{i}$ is the ground reaction force at the foot of leg $i$ with respect to $G_{0}$ denoted by vector $\left[F_{i x} F_{i y} F_{i z}\right]^{T}, r_{i}$ is the location vector of the foot tip $P_{i 3}$ with respect to point $\mathrm{P}_{0}$ at any instant, $M_{0}$ is the vector of actuator torques acting on the trunk body and payload (combined) denoted by $\left[\begin{array}{lll}0 & 0 & \sum_{i=1}^{6} M_{i 1}\end{array}\right]^{T}$.

The next set of eighteen equations of (22) defines the relationship between the actuator torques and ground reaction forces on the legs such that for leg $i$,

$$
M_{i}=-B_{i}^{-1}\left(A_{i} F_{i}+M_{e i}\right) \in R^{3 \times 1}
$$

where $M_{i}$ is the vector of actuator torques of leg $i$ denoted by 
$\left[\begin{array}{lll}M_{i 1} & M_{i 2} & M_{i 3}\end{array}\right]^{T} ; M_{e i}$ is the vector representing the coriolis, centripetal, gravitational forces and moments acting on the

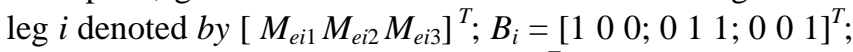
$A_{i}=\left[r_{i 1 x} r_{i 1 y} r_{i 1 z} ; r_{i 2 x} r_{i 2 y} r_{i 2 z} ; r_{i 3 x} r_{i 3 y} r_{i 3 z}\right]^{T}$.

\section{Optimization of Power Consumption}

Equations, (26), (27) and (28) reveal that the number of unknowns $(n=36)$ is more than that of constraint equations $(m=24)$. Hence, there is no unique solution to these equations. The solution to the problem is to be obtained by optimization through linear programming i.e., minimizing the total instantaneous power consumption $\left(P_{i n}=M_{i j} \omega_{i j}\right)$ of the system, which is considered as the objective function with respect to linear equality and inequality constraints. Therefore, optimization problem can be stated as follows:

Minimize

$$
\left.P_{\text {in }}=\int \sum_{i=1}^{6} \sum_{j=1}^{3} P_{i j}(t) d t\right)
$$

Subject to some (a) inequality and (b) equality constraints, as given below.

\section{1) Inequality constraints}

a) Joint torque inequality constraints

The actuators selected for the hexapod has torque limitations, such that,

$$
M_{i j, \min } \leq M_{i j} \leq M_{i j, \max } \text { for } i=1 \text { to } 6, j=1 \text { to } 3
$$

where $M_{i j}$ is the torque at the $j^{\text {th }}$ joint of $i^{\text {th }} \operatorname{leg}, M_{i j, \min }$ and $M_{i j, \max }$ are the limits of torque at the $j^{\text {th }}$ joint of $i^{\text {th }}$ leg, which are generally dependent on the motor specifications.

\section{b) Friction inequality constraints}

In the present study, it is assumed that there is no slippage of the foot due to the limiting angle between resultant foot force and normal to the ground at the point of contact, and that only static coefficient of friction exists. Hence,

$$
\left|F_{i x y}\right|=\sqrt{ }\left(F_{i x}^{2}+F_{i y}^{2}\right) \leq \mu_{i} F_{i z}
$$

where $\mu_{i}$ is the static friction coefficient between the foot of leg $i$ and ground i.e., at interface during the support phase. This nonlinear inequality corresponds to a friction cone [20]. Further, the friction cone can be linearized into a friction pyramid [24]-[25] that lie within the cones, such that the $4 i$ inequality constraints of (32) are sufficient for the formulation and implies (31).

$$
\begin{aligned}
& F_{i x}+\mu_{\text {eff }} F_{i z} \geq 0 \\
& -F_{i x}+\mu_{e f f} F_{i z} \geq 0 \\
& F_{i y}+\mu_{e f f} F_{i z} \geq 0 \\
& -F_{i y}+\mu_{e f f} F_{i z} \geq 0
\end{aligned}
$$

where $\mu_{\text {eff }}=\mu_{i} / \sqrt{ } 2$

\section{c) Normal force inequality}

Since the feet of the hexapod cannot grasp the ground, only positive vertical forces that can be exerted by the feet on the ground are considered. Therefore,

$$
F_{i z} \geq 0
$$

\section{2) Equality constraints}

Equations (26) and (27) are the taken as the equality constraints. Further, to let the support legs have a stable contact with the ground, the moments applied by the legs should be equal to that of the weight of the system acting at the center of gravity of the robot. So, this additional equality constraint can expressed as follows:

$$
\sum \tilde{r}_{P_{i 3} O}^{G_{0}} \times F_{i}=\tilde{r}_{C_{m} O}^{G_{0}} \times F_{g}
$$

where $\tilde{r}_{P_{i 3} O}^{G_{0}}$ and $\tilde{r}_{C_{m} O}^{G_{0}}$ are the skew symmetric matrix of the location vectors of the foot tip $P_{i 3}$ and center of mass of the whole system, respectively, with respect to origin $O$ in frame $G_{0} ; F_{g}$ is the vector of gravitational forces acting on the system denoted by $\left[\begin{array}{llll}0 & 0 & m_{\text {total }} \cdot g\end{array}\right]^{T}$.

\section{NUMERICAL ILLUSTRATION}

To determine the efficacy of the model, simulation of the walking robot with wave-crab gait $(D F=1 / 2)$ on a flat terrain are carried out in the present study. The robot moves in transverse direction from left to right with a positive crab angle $\left(\theta_{c}\right)$ of 80 degrees. Here, the $X$ and $Y$-translational velocity components of the trunk body are not independent unlike straight-forward motion. Therefore, the translational velocity components of the trunk body with respect to global frame $G$ are assumed to follow the relationship:

$$
\dot{r}_{P_{0} O}^{G}=\left(\dot{x}_{P_{0} O}^{G}, \dot{x}_{P_{0} O}^{G} \cdot \cot \theta_{c}, 0\right)^{T}
$$

The kinematic analysis of the hexapod robot is carried out followed by dynamic analysis. At time $t=0$, the position and orientation of $\mathrm{P}_{0}$ with respect to global frame $G$ are given by $p_{0}^{G}=\{0,0.45,0.15,0,0,0\}^{T}$. Considering all the initial velocity components (both translational and angular) as zero, the maximum translational reached by the trunk body are assumed to be $\dot{x}_{P_{0} O}^{G}=0.005 \mathrm{~m} / \mathrm{s}$. In addition to the above, the other necessary inputs are $\eta_{G}=(0,0,0)^{T}, \theta_{i 1}= \pm\left(90^{0}-\theta_{c}\right)$, $\beta_{i 2}= \pm 16^{0}, \beta_{i 3}= \pm 69^{0}$ (for $i=1$ to 6 ), maximum height of swing along $\mathrm{Z}$ with respect to $G$ is $0.015 \mathrm{~m}$.

The simulations are run for three duty cycles $n=3$, body stroke $s_{0}=0.03 \mathrm{~m}$ and time step $h=0.1 \mathrm{~s}$ in a commercially available compiler MATLAB [26]. Once kinematic analysis results are obtained, the relevant motion parameters of the trunk body and leg tip are provided as inputs to the inverse dynamic analysis code in MATLAB. The optimization problem formulated here is solved using linear programming 
based on interior point method. The coefficient of friction $\mu_{i}=0.3$ is considered at the foot ground interface, while the torque limits of the motors are $\pm 6 \mathrm{Nm}$. The computed results of the optimal force distribution are shown in Fig. 4 over three gait cycles with total cycle time 12.4s. The normal feet-force component $F_{i z}$ of all the six legs of the hexapod are plotted against time at an interval of $h=0.1 \mathrm{~s}$ as shown in Fig. 4. Since in a wave-crab tripod gait the DF is $1 / 2$, the support and swing phase times are equal for all the legs. The tripod gait is composed of the following sequences: 1) legs 1-4-5 in support phase, legs 2-3-6 in swing phase; 2) legs 2-3-6 in support phase, legs 1-4-5 in swing phase (refer Fig. 1 for the leg numbering sequence).

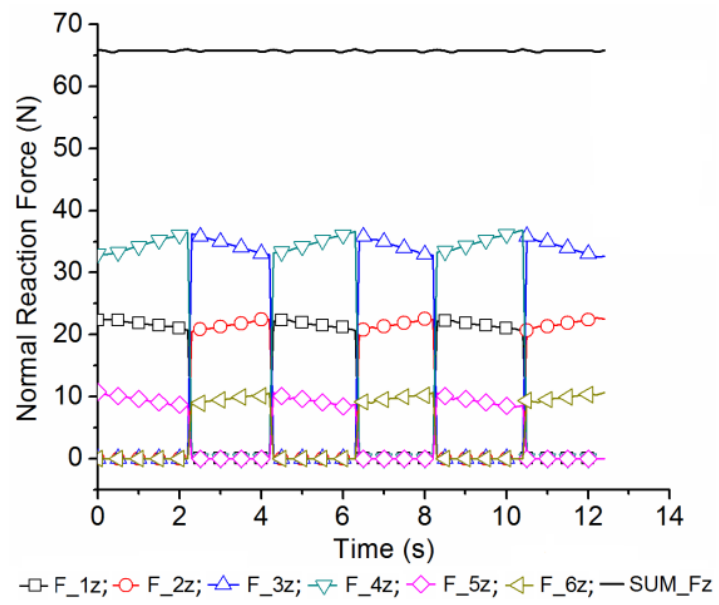

Fig. 4. Normal force distribution in the legs with respect to frame $G_{0}$.

From Fig. 4, it is observed that as the hexapod moves with wave-crab gait in transverse direction (left to right), the feet-force patterns of the legs 1-2, 3-4 and 5-6 forms a wave like pattern. As expected, the normal forces in legs 1-3-5 have a descending trend while those in legs 2-4-6 have an ascending trend. It happens so, due to the transverse motion of the trunk body from left to right. In other words, as the trunk body moves along the transverse direction, the entire center of gravity of the hexapod also moves in that direction. Hence, during support phase the feet forces on the legs 1-3-5 (odd numbered legs) will decrease while those on the legs 2-4-6 (even numbered legs) will increase with time till the start of swing phase. When the legs are lifted during swing phase, the forces on leg tips are zero and denoted by straight line.

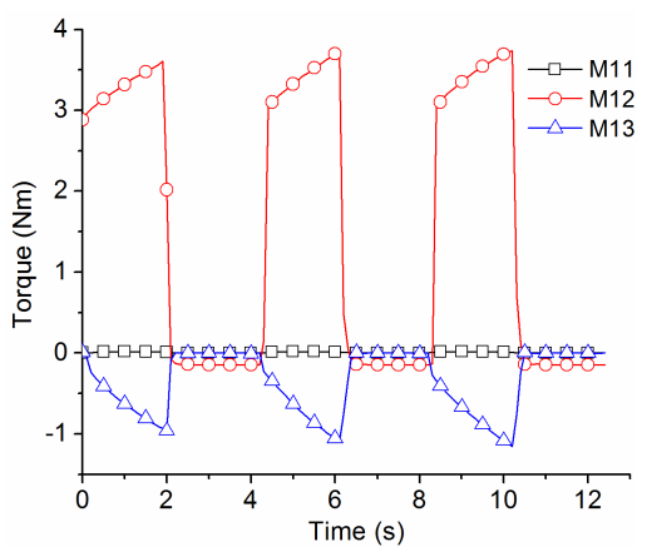

Fig. 5. Joint torque distribution in leg 1.
Another observance is that the summation of all the foot forces along z-direction with respect to $G_{0}$ balances the weight of the hexapod $(65.7 \mathrm{~N})$ at any instant of time (Fig. 4). Also in Fig. 5, one can easily observe that in leg 1, the joint torques of joint 2 and 3 in support phase take significant values, while in swing phase are close to zero. The joint torque values of joint 1 of leg 1 are very small since the crab angle (with respect to joint 1) is constant and theoretically it has zero angular velocity. It is also observed that the joint 2 experiences maximum torque at instant of time.

The variation of instantaneous power $\left(P_{i n}\right)$ consumption throughout a locomotive cycle of the hexapod is as shown in Fig. 6. The highest peak arises at the time, when the swing and support legs change stance.

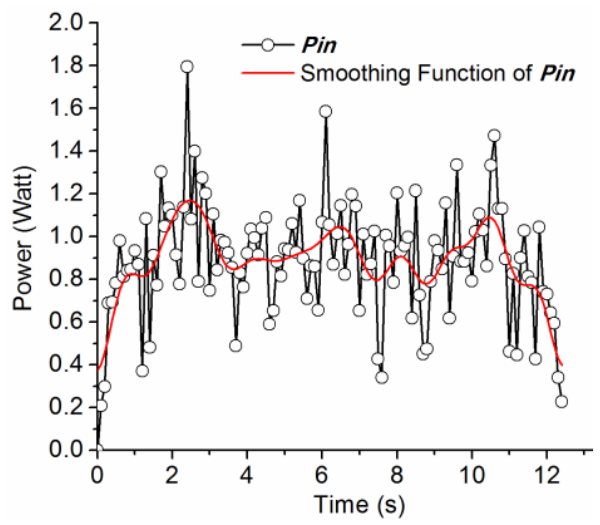

Fig. 6. Variation of total power consumption by the joints of all the legs.

\section{CONCLUSION}

In the present study, the inverse dynamic and power consumption model of the wave-crab gait for a hexapod robot is developed as constrained optimization problem. The inverse dynamics model takes into consideration the inertia effects of the swing legs on the trunk body and the support legs. Also, the issue of the optimal foot forces distribution from the view point of minimization of the instantaneous power consumption is addressed in the paper. A computationally efficient solution for the equilibrium force and moment fields is derived. The study reveals that for a wave -crab gait with $\mathrm{DF}=1 / 2$, the total power consumption is maximum when the legs changes stance (support to swing or swing to support). Future study in the area will focus on the relationship of energy efficiency with different gait patterns and walking speed. The real time implementation of the model in robot control will also be addressed in the future scope of work.

\section{ACKNOWLEDGMENT}

The second author is thankful to Director, National Institute of Technology, Durgapur, for his kind permission to publish this paper.

\section{REFERENCES}

[1] S. M. Song and K. J. Waldron, Machines That Walk: The Adaptive Suspension Vehicle, The MIT Press, Cambridge, Massachusetts, 1989.

[2] P. G. de Santos, E. Garcia, and J. Estremera, Quadrupedal Locomotion-An Introduction to the Control of Four-legged Robots, London: Springer-Verlag, 2006, ch. 6. 
[3] X. Y. S. Castro, M. G. Murillo, L. A. P. Resendiz, and E. C. Castañeda, "Kinematics of hex-Piderix - a six-legged robot - using screw theory," International Journal of Advanced Robotic Systems, vol. 10, no. 19, pp. $1-8,2013$.

[4] S. S. Roy and D. K. Pratihar, "Kinematics, dynamics and power consumption analyses for turning motion of a six-legged robot," Journal of Intelligent and Robotic Systems, vol. 74, no. 3-4, pp. 663-668, 2014.

[5] M. Akdag, H. Karagülle, and L. Malgaca, "An integrated approach for simulation of mechatronic systems applied to a hexapod robot," Mathematics and Computers in Simulation, vol. 82, pp. 818-835, 2012.

[6] J. Denavit and R. S. Hartenberg, "A kinematic notation for lower-pair mechanisms based on matrices," Trans ASME J Applied Mech, vol. 23, pp. 215-221, 1955.

[7] Z. Y. Wang, X. L. Ding, A. Rovetta, and A. Giusti, "Mobility analysis of the typical gait of a radial symmetrical six-legged robot," Mechatronics, vol. 21, no. 7, pp. 1133-1146, 2011.

[8] C. R. Rocha, C. P. Tonetto, and A. Dias, "A comparison between the Denavit-Hartenberg and the srew-based methods used in kinematic modeling of robot manipulators," Robotics and Computer Integrated Manufacturing, vol. 27, pp. 723-728, 2011

[9] C. L. Bottasso, A. Croce, L. Ghezzi, and P. Faure, "On the solution of inverse dynamics and trajectory optimization problems for multibody systems," Multibody System Dynamics, vol. 11, pp. 1-22, 2004.

[10] D. M. Gorinevsky and A. Y. Shneider, "Force control in locomotion of legged vehicles over rigid and soft surfaces," Int. J. Robotic Research, vol. 9, no. 2, pp. 4-23, 1990.

[11] J. Furusho and A. Sano, "Sensor-based control of a nine-link biped," Int. J. Robotic Research, vol. 9, no. 2, pp. 83-98, 1990.

[12] J.-S. Chen, F.-T. Cheng, K.-T. Yang, F.-C. Kung, and Y.-Y. Sun, "Optimal force distribution in multilegged vehicles," Robotica, vol. 17, pp. 159-172, 1999

[13] S. S. Roy, A. K. Singh, and D. K. Pratihar, "Estimation of optimal feet forces and joint torques for on-line control of six-legged robot," Robotics and Computer-Intergrated Manufacturing, vol. 27, no. 5, pp. 910-917, 2011

[14] S. S. Roy and D. K. Pratihar, "Dynamic modeling and energy consumption analysis of crab walking of a six-legged robot," in Proc. IEEE Conference on Technologies for Practical Robot Applications (TePRA), 11-12 April 2011, pp. 82-87.

[15] B. Jin, C. Chen, and W. Li, "Power consumption optimization for a hexapod walking robot," Journal of Intelligent and Robotic Systems, vol. 71, no. 2, pp. 195-209, August 2013

[16] W. Y. Jiang, A. M. Liu, and D. Howard, "Optimization of legged robot locomotion by control of foot-force distribution," Transactions of the Institute of Measurement and Control, vol. 26, no. 4, pp. 311-323, 2004.

[17] M. S. Erden and K. Leblebicioglu, "Torque distribution in a six-legged robot," IEEE Trans. on Robotics, vol. 23, no. 1, pp. 179-186, 2007.

[18] K. Li, X. Ding, and M. Ceccarell, "A total torque index for dynamic performance evaluation of a radial symmetric six-legged robot," Front. Mech. Eng., vol. 7, no. 2, pp. 219-230, 2012.

[19] D. Zhou, K. H. Low, and T. Zielinska, "An efficient foot-force distribution algorithm for quadruped walking robots," Robotica, vol 18 , pp. 403-413, 2000

[20] J. T. Lei, "Energy efficiency analysis of quadruped robot with trot gait and combined cycloid foot trajectory," Chinese Journal of Mechanical Engineering, vol. 27, no. 1, pp. 138-145, 2014.

[21] H. Hahn, Rigid Body Dynamics of Mechanisms 2, $1^{\text {st }}$ ed., Berlin-Heidelberg: Springer-Verlag, 2003, p. 61.

[22] Basic ADAMS full Simulation Training Guide, Ver. 12.0, Ann Arbor, USA: Mechanical Dynamics, 2002, pp. 27-61.

[23] M. Bennani and F. Giri, "Dynamic modelling of a four-legged robot," Journal of Intelligent and Robotic Systems, vol. 17, no. 4, pp. 419-428, December 1996.

[24] L. S. M. Filho and R. Prajoux, "Locomotion control of a fourlegged robot embedding real-time reasoning in the force distribution," Robot. Auton. Syst., vol. 32, pp. 219-235, 2000.

[25] J. A. Galvez, J. Estremera, and P. G. de Santos, "A new legged robot configuration for research in force distribution," Mechatronics, vol. 13, pp. 907-932, 2003

[26] W. Y. Yang, W. Cao, T.-S. Chung, and J. Morris, Applied Numerical Methods Using Matlab, Hoboken, USA: John Wiley \& Sons, 2005, ch. 7.

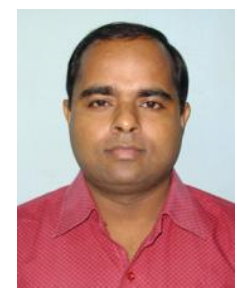

Abhijit Mahapatra received his B.E and M.Tech degrees in mechanical engineering from B.E. College (currently IIEST Shibpur), Shibpur and NIT, Durgapur in 2002 and 2008, respectively. Currently, he is working as a senior scientist in Virtual Prototyping and Immersive Visualization Lab., CSIRCentral Mechanical Engineering Research Institute, Durgapur, India. He has published number of research papers in national and international journals, conference proceedings and filed a number of patents in area of product development. His current research interests include design \& analysis, multi-body dynamics, modeling and simulation of legged robots.

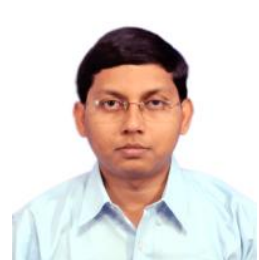

Shibendu Shekhar Roy received his B.E and M.Tech degrees in mechanical engineering from R.E. College, Durgapur (Presently, NIT, Durgapur) in 1999 and 2001, respectively. He obtained his Ph.D from IIT, Kharagpur, India, in 2011 Currently, $\mathrm{He}$ is an associate professor in the Department of Mechanical Engineering, National Institute of Technology, Durgapur, India. From March 2001 to December 2006 he was a scientist at the Central Mechanical Engineering Research Institute, Durgapur, CSIR, India. He has published a great deal of research papers at national and international journals, conference proceedings, book chapters and filed number of patents in product development. He has written a book on "Modeling and analysis of six-legged robots", which has been published by Lap Lambert Academic Publishing GmbH \& Co. KG, Germany. His current research interests include application of computational intelligent tools like fuzzy logic, genetic algorithm, Neural networks etc. for modelling of different manufacturing process and robotic systems.

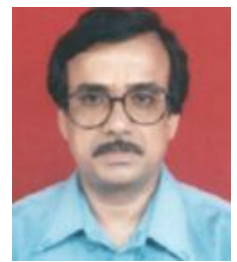

Dilip Kumar Pratihar received his B.E. (Hons.), M.Tech., Ph.D. and he received Ph.D. from IIT Kanpur, India, in the year 2000. Besides several scholarships and best paper awards, he received the University Gold Medal for securing the highest marks in the University in 1988, the A.M. Das Memorial Medal in 1987, the institution of engineers' medal in 2002, and others. He completed his post-doctoral studies in Japan (6 months) and Germany (1 year) under the Alexander von Humboldt Fellowship Programme. He is currently working as a professor in the Department of Mechanical Engineering, IIT Kharagpur, India. His research areas include robotics, soft computing and manufacturing science. He has published more than 135 papers in different journals and conference proceedings. He has written a textbook on "Soft Computing", which has been published by Narosa Publishing House, New Delhi and Alpha Science International Publisher, UK. Recently, this book has been translated into Chinese. He has edited a book on "Intelligent and Autonomous Systems", which was in 2010 published by Springer-Verlag, Germany. He has been included as a member of the program committee for several international conferences. He has been selected as the editorial board member of eight international journals. He has been elected as a fellow of the Institution of Engineers (I) and a member of IEEE. 